\title{
LOS FENÓMENOS CELESTES EN COSTA RICA: AEROLITOS, BÓLIDOS Y LLUVIAS DE ESTRELLAS (1799-1910)
}

\author{
Flora J. Solano Chaves ${ }^{1 *}$, Ronald Díaz Bolaños ${ }^{2} \&$ Mario Fernández Arce ${ }^{1,3}$ \\ ${ }^{1}$ Centro de Investigaciones Geofísicas (CIGEFI), Universidad de Costa Rica. \\ ${ }^{2}$ Escuela de Historia y Centro de Investigaciones Geofísicas, \\ Universidad de Costa Rica. \\ ${ }^{3}$ Centro Sismológico de América Central, Universidad de Costa Rica. \\ *Autor para contacto: solanofj04@yahoo.com
}

(Recibido: 16/02/07; aceptado: 01/11/07)

\begin{abstract}
Data collection of astronomical phenomena in Costa Rica began at the end of the colonial period (1799). Most of this information reveals the social impact that they caused in the region. Scholars of that time tried to find a rational explanation for those phenomena, especially meteor showers and meteorites, in order to contrast the religious conceptions. This paper analyzes the most representative data obtained from the observations during the period 1799-1910.

Key words: Costa Rica, meteorites, aerolites, fireballs, meteor showers, social impact.

RESUMEN: Los registros de observaciones de fenómenos astronómicos en Costa Rica se remontan a finales de la época colonial (1799). Estos detallan el impacto que tuvieron en la sociedad del momento, principalmente las lluvias de estrellas y la caída de meteoritos. Desde esa época se ha buscado una explicación racional a dichos eventos ante la alternativa conceptual religiosa imperante. El presente trabajo resume y analiza los datos más representativos obtenidos en dichas observaciones entre 1799 y 1910.

Palabras clave: Costa Rica, meteoritos, aerolitos, bólidos, lluvias de estrellas, impacto social.
\end{abstract}




\section{INTRODUCCIÓN}

Documentos con detalles de algunos acontecimientos histórico-científicos relevantes relacionados con la observación de fenómenos celestes entre 1799 y 1910, concernientes con lluvias de estrellas, aerolitos y bólidos en los cielos costarricenses fueron rescatados y escudriñados para la elaboración de este trabajo.

Se inicia con una recapitulación de los antecedentes histórico-científicos en Europa y América desde finales del siglo XVIII y comienzos del XIX, en los que se aborda el contexto de la época y una aproximación conceptual de los fenómenos celestes. Se visualizan las observaciones más sobresalientes realizadas en Costa Rica referentes a las lluvias de estrellas de 1799 y 1833 , la caída de un meteorito en 1857, la observación de estrellas fugaces en 1896 y de bólidos en 1896 y 1910 que se presentan en la figura 1 (cf. Soto, 2004). Se interpreta la reacción y actitud de la sociedad de la época, enfatizando el interés científico que despertaron tales manifestaciones.

\section{ASPECTOS CONCEPTUALES HISTÓRICO-CIENTÍFICOS}

La primeras observaciones de lluvias de estrellas (Hardy et al., 1983) se endosan a los primeros registros elaborados por los pobladores de China en la Antigüedad. No obstante, estos fenómenos empezaron a despertar interés científico en 1803, cuando la Academia Francesa decidió emprender estudios sobre meteoritos en forma sistemática (De Bon \& Carvajal, 2004).

En el siglo XIX, el concepto de meteorito era sinónimo de aerolito: un pedazo desprendido de un bólido o "piedra emanada de las alturas de la atmósfera". El término de bólido designaba todo objeto luminoso que estallaba al entrar en contacto con la atmósfera terrestre y se fragmentaban. En ese entonces, se creía que los bólidos integraban el Sistema Solar, asociados a los "planetas de cortísimas dimensiones" o asteroides recién descubiertos. Se consideró su procedencia de erupciones volcánicas de la Luna, tesis compartida por Heinrich Olbers (1758-1840), Pierre de
Laplace (1749-1827), Joseph Lagrange (17361813), Siméon Poisson (1781-1840) y JeanBaptiste Biot (1774-1862), mientras que Ernst Chladni (1756-1827), en 1794 los asociaba con cuerpos que vagaban libremente por el espacio y para Gabriel Daubrée (1814-1896), en 1870, tan solo reductos de planetas extintos (Flammarion, 1883; De Bon \& Carvajal, 2004).

La importancia de los meteoritos para la ciencia del siglo XIX estribaba en el análisis de los elementos componentes de cuerpos extraterrestres. Este interés puntual permitió la creación de colecciones, entre las que se destacan las del Museo de Historia Natural de París, el Museo Británico de Londres y el Museo Mineralógico de Viena (Flammarion, 1883).

En el presente, la ciencia atribuye el origen de los meteoritos a cuerpos cósmicos desprendidos de algún objeto mayor en el espacio. Sus tamaños varían desde un fino polvo hasta grandes bloques $>1 \mathrm{~km}$ de diámetro, y cae continuamente sobre la Tierra, por lo que el planeta está sometido a un continuo bombardeo de fragmentos rocosos procedentes del espacio exterior.

En el siglo XIX, se determinó que los meteoritos estaban constituidos por minerales enfriados tras impactar el suelo terrestre y se les describía por su forma poliédrica imperfecta, cubiertos de una capa negra y reluciente con un espesor de un milímetro (Flammarion, 1883). La ciencia actual ha revelado su composición química y mineralógica, consistente esencialmente en aleaciones de hierro-níquel, de silicatos como olivino o piroxenos, o una mezcla de ambos. Además se han encontrado aminoácidos y compuestos orgánicos, lo que indica la existencia de materia orgánica en el espacio exterior.

En el siglo XIX, la ciencia conceptualizaba a las estrellas fugaces o errantes como fenómenos de una naturaleza similar a la de los meteoritos, definiéndolas como: "estrella desprendida de la esfera celeste que se apaga sin ruido". Se calculó su altura en unos $120 \mathrm{~km}$ al inicio de la observación y $80 \mathrm{~km}$ al extinguirse y una velocidad estimada entre los 12 y $70 \mathrm{~km}$ por segundo (Flammarion, 1883).

Camille Flammarion, astrónomo francés, fundador del observatorio astronómico de Juvisysur-Orge y de la Sociedad Astronómica de Francia 


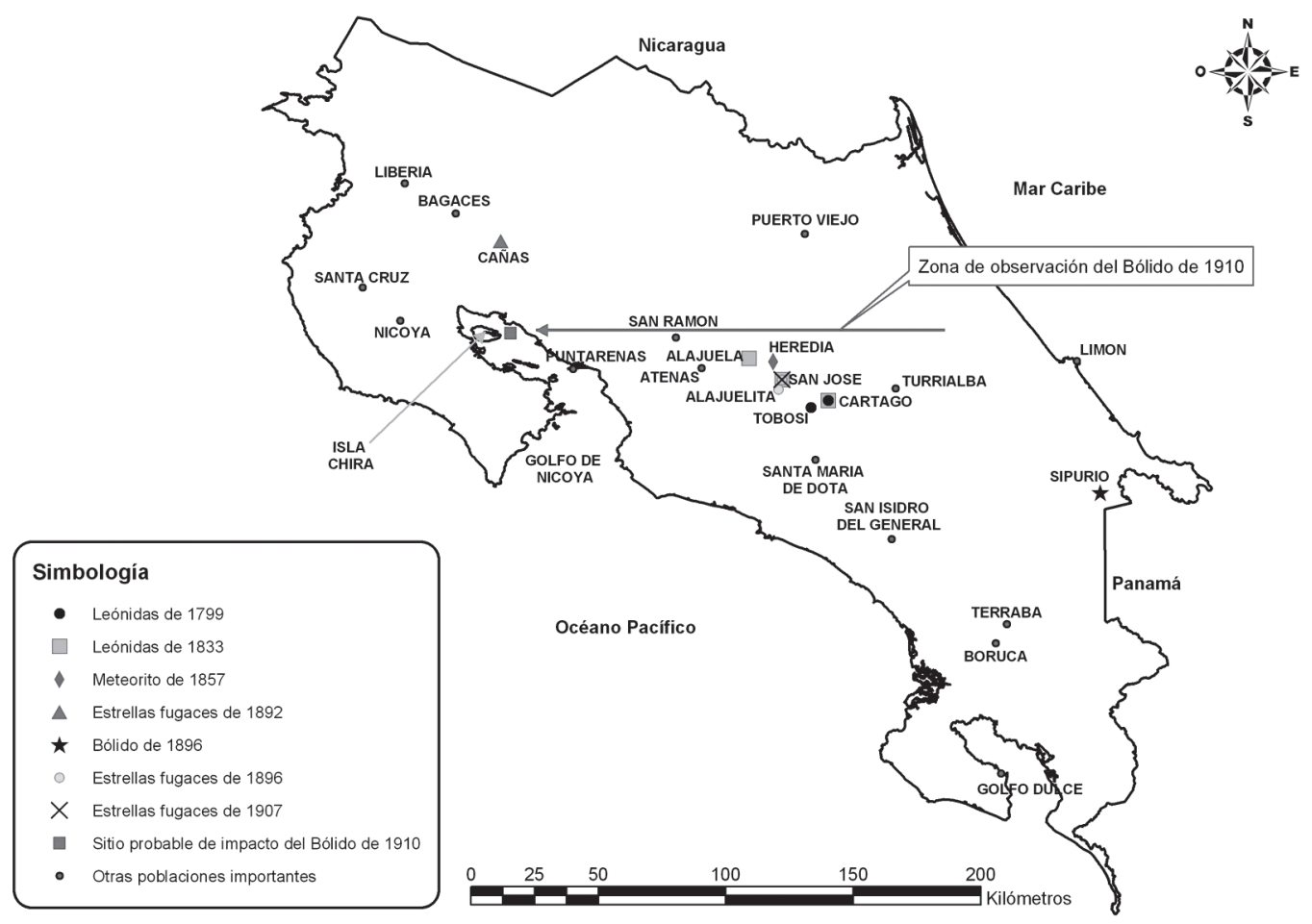

Fig. 1: Fenómenos celestes observados en Costa Rica (1799 - 1910).

y autor de varias publicaciones científicas relacionadas con la astronomía, explicó el origen de las estrellas fugaces como producto del "efecto al choque sucesivo de la tierra con una porción de corpúsculos que circulan en los espacios celestes, y que vienen a nosotros con velocidades próximamente iguales entre sî" (Flammarion, 1883). En este sentido, las lluvias de estrellas no tenían un origen distinto, sino que procedían de determinadas zonas del cielo.

\section{EL DESARROLLO DE LA CIENCIA LATINOAMERICANA EN EL SIGLO XVIII Y SUS REPERCUSIONES EN COSTA RICA}

Durante el siglo XVIII se desató un vivo interés por el comportamiento del ser humano ante las variaciones de la naturaleza. Es la fase del movimiento ilustrado que promueve reformas y progreso, es el período de la creación de academias de ciencias, entre ellas, la Academia de las Artes y las Ciencias en América, la Asociación Británica para el Desarrollo de la Ciencia, la Asociación Americana para el Desarrollo de la Ciencia y la Asociación Francesa para el Desarrollo de la Ciencia, favoreciendo estas instituciones la divulgación científica.

Proliferaron las expediciones científicas en las actividades de medición de límites, construcción de fortificaciones y observaciones astronómicas. Entre ellas, las de Charles-Marie de $\mathrm{La}$ Condamine en Quito (1736), la de Hipólito Ruiz y Luis Pabón en el Virreinato de Perú de 1777 a 1788; la de Martín Sessé y Lacaste y José María Mociño en Nueva España de 1788 a 1803; la expedición naval de Alejandro Malaspina de 1789 a 1794, la de Alexander von Humboldt y Aimé Bonpland (1778-1858), entre 1799 y 1804, a la América española, cuyas hazañas marcaron un hito en la historia científica regional y universal. De interés para este trabajo es destacar las investigaciones de Alejandro Malaspina, que dentro de su tripulación y según lo asevera Font-Tullot 
(1986) traía astrónomos. Por otro lado se puntualiza la labor del naturalista von Humboldt, uno de los artífices de la geografía moderna, quien realizó observaciones meteorológicas y astronómicas, explora sobre los meteoritos (1799) y el tránsito de Mercurio frente al disco solar (1802).

De acuerdo con Meléndez (1974), Centroamérica se caracterizó por una fase plena de avances y enseñanzas gracias al explosivo ambiente ilustrado, que se difunde en la Universidad de San Carlos de Guatemala y los seminarios conciliares. Destaca la obra "Astronomía Práctica o Compendio de 84 tablas astronómicas", del guatemalteco Joaquín Manuel Calderón de la Barca (1743), “Tablas Astronómicas” del presbítero Juan José Padilla (¿1750?) y la del célebre cura costarricense José Antonio de Liendo y Goicoechea, un adelantado en el estudio de las ciencias físicas que enseñaba, como en el caso de la astronomía, con materiales que había traído de España. Este sacerdote difundía sus conocimientos a través de "La Gazeta de Ghoatemala".

Otro de los pilares sobre los que se cimentó la ciencia centroamericana, es el hondureño Dr. José Cecilio del Valle, hombre de grandes luces, que estudió en la Universidad de San Carlos de Guatemala, donde la influencia intelectual de Liendo y Goicoechea caló hondo en su formación humanista y científica (Solano, 1999).

Valle insta entre 1826 y 1827 al astrónomo y difusor de la emancipación mexicana Don Manuel de Mier y Terán que en el viaje de reconocimiento que haría de Nuevo México llevara un registro de las manifestaciones de la naturaleza. Su interés por la difusión de la cultura americana y los estrechos contactos que estableciera con estudiosos del conocimiento científico en Europa y América, entre ellos Bonpland y von Humboldt, lo conducen (Leiva, 1980) a solicitar públicamente en 1824 que se organice una expedición científica y apenas se informa del segundo viaje de Humboldt, le escribe invitándole a que lo extienda hasta Centro América (Leiva, 1984).

El siglo XIX experimenta acontecimientos histórico-científicos que cristalizan en una era expansionista europea, cuya esfera de influencia abarcó a muchas naciones. A principios del siglo XIX, Costa Rica se revitalizó gracias a cambios en su economía y los aires ilustrados se afianzan, era una zona marginal de la Capitanía General de Guatemala, de escasos recursos económicos. La provincia fue visitada desde el siglo XVII por personajes de diversas culturas, interesados en el comercio y otros con orientaciones más científicas, caso del navegante inglés George Vancouver, compañero de viaje en varias oportunidades del eminente explorador James Cook. Vancouver realizó el primer levantamiento de las costas en Isla del Coco. Sus datos fueron utilizados años más tarde por Henri Pittier en sus investigaciones sobre la isla (González Flores, 1976).

En los años previos a la independencia se crearon centros de pensamiento como la Casa de Enseñanza de Santo Tomás en San José (1814), origen de la universidad homónima (1843).

\section{LA LLUVIA DE ESTRELLAS DE NOVIEMBRE DE 1799}

El 20 de noviembre de 1799, el gobernador don Tomás de Acosta redacta un informe al Capitán General Domás y Valle, de la Capitanía General en la Ciudad de Guatemala, acerca de la primera observación documentada de un fenómeno celeste en Costa Rica entre el 11 y 12 de noviembre de ese mismo año, advirtiendo que era "uno de los más portentosos fenómenos que se han visto en este hemisferio y tal vez en el mundo" (ver Anexo 1). El documento que describe la "Lluvia de fuego", puntualiza aspectos meteorológicos, hechos observados y la reacción del pueblo. Las observaciones y análisis sobre el fenómeno de 1799 que acaparó la atención de los residentes de Cartago, también fueron observados y documentados para la región de Tobosi, uno de los pueblos de indios que rodeaban la capital colonial de la provincia de Costa Rica.

El Gobernador asoció el hecho con un fenómeno natural ligado a los efectos que provocan los volcanes cercanos (Cartago, Turrialba, Dragón) y a muchos minerales que existen en los contornos. El señor Acosta y en vista de que las ciudades estaban cerca de los volcanes envía a personas de "satisfacción que inmediatamente fueran á reconocerlos con prolijo examen para ver si humeban, si se les advertía alguna inflamación, si tenían alguna grieta, si se les oía algún susurro, 
si exhalaban algún olor sulfúreo o bituminoso, ó si se encontraba en ellos novedad particular". El gobernador pensó que cualquiera que fuera la causa de "estos efluvios inflamables, es muy de temer una erupción, porque en vista de la abundancia que hubo de ellos y lo cargado que estaban de materia eléctrica, considero que el paraje que los arrojó, sea el que fuere, sobreabunda de aquella materia, y por consiguiente muy expuesto a reventar". Afirmó que "Yo puedo estar muy errado en mi juicio, porque no he hecho estudios de física experimental, pero de todos modos creo de mi obligación participar á Usted lo acaecido...por si hubiere alguna cosa que prevenir, y asegurarle al mismo tiempo que no hay lengua, pluma ni pincel capaz de pintar debidamente lo que sucedió la noche del once al doce del corriente, y que la expresión de que llovió fuego sólo se aparta de la realidad en que no cayó en tierra todo el que se desprendió de la atmósfera".

Las ideas científicas que circundaban el período colonial y los rudimentos en este campo que manejaba el gobernador Acosta le indujeron a percibir la "lluvia de fuego" de 1799 como un fenómeno natural, no así asociándolo a una explicación religiosa que hubiera sido lo usual en la época. Atribuyó su génesis a la actividad del volcán Irazú, que había hecho una portentosa erupción en 1723, según consta en los informes de su predecesor Diego de La Haya Fernández y al Cerro Dragón o Caraigres, considerado volcán por los residentes de la provincia por su forma similar a un cono volcánico (Chacón, 1967).

El aporte más significativo es legar un registro detallado del fenómeno de la lluvia de estrellas con base en la observación y el recuento de datos, pese a que sus descripciones contienen elementos de exageración (Soto, 2004). Las descripciones se hicieron con base en el conocimiento de la época haciendo alusión a la Física Experimental, disciplina que había sido introducida durante la reforma impulsada por Fray José Antonio de Liendo y Goicoechea en la Universidad de San Carlos de Guatemala al finalizar la década de 1760 (Solano, 1999).

El acontecimiento narrado por el gobernador Tomás de Acosta en Costa Rica tiene similitud con la descripción hecha por Bonpland y Humboldt en Cumaná (Venezuela) el 12 de noviembre de 1799 y descrito por este último de la siguiente forma (Pereira, s.f.; Soto, 2004): "Media hora después de la dos de la mañana, los más extraordinarios y luminosos meteoros fueron vistos en dirección de M. Bonpland, que se había levantado para disfrutar del aire fresco, los percibió primero. Miles de bólidos y estrellas fugaces se sucedieron unas a otras durante el espacio de cuatro horas. Sus direcciones eran muy regulares de norte a sur. Llenaban un espacio en el cielo que se extendía exactamente $30^{\circ}$ de norte a sur. En una amplitud de $60^{\circ}$ los meteoros fueron vistos salir sobre el horizonte al ENE y al E., para describir arcos más o menos extendidos, y caer hacia el sur, después de haber seguido la dirección del meridiano. Algunos de ellos alcanzaron una altura de $40^{\circ}$, y todos excedían los $25^{\circ}$ o $30^{\circ}$. M Bonpland. Estableció que, desde la primera aparición del fenómeno, no había en el firmamento un espacio igual en extensión a tres diámetros de la Luna, que no estuviera lleno en cada instante de bólidos y estrellas."

Flammarion (1883), describió las observaciones de Bonpland y Humboldt como sigue: "En el mes de Noviembre de 1799, y en su noche del 11 al 12 A. de Humboldt y Bonnpland [sic] asistieron en Cumana [sic] (América) á un verdadero chaparrón de estrellas fugaces. Bonnpland [sic] declara que no había en el cielo un espacio cuya estension [sic] fuese igual á tres diámetros de la luna que no se viera á cada momento lleno de estrellas fugaces. Los habitantes de Cumana estaban aterrorizados con el fenómeno: los mas ancianos recordaban que en 1766 se habia presenciado otro análogo, acompañado de un temblor de tierra."

Esta actividad cósmica también fue vivida por otros habitantes en la misma región de América del Sur. Gonzalo Pereira (s.f.), enfatiza que la presencia celeste causó tal estupor en los pobladores guaraníes (Kereimba), que horrorizados ante tan descomunal evento, frenaron el ataque que tenían perpetrado contra el fuerte Saipurú, bajo control español. Este recinto ocupaba un punto estratégico que había utilizado tanto por los incas como por los conquistadores contra el continuo asedio guaraní.

El recuerdo de la lluvia de estrellas de finales del siglo XVIII, vista en numerosos puntos del continente americano y también en Europa (Soto, 2004), caló, según unos, en la memoria colectiva de los costarricenses, como propone el escritor 
Máximo Soto Hall en "Un vistazo sobre Costa Rica en el siglo XIX" (ver Paéz, 1994): "Un aspecto astronómico relevante en ese inicio del siglo es la lluvia de estrellas en la noche del 12 al 13 de noviembre de 1800 [sic]." Otros difieren, como Páez (1994), para quien dicho "fenómeno celeste parece no haber motivado a ninguno de nuestros antepasados, ya no por la astronomía, sino por las ciencias en general".

Para esa misma fecha y en vista de que no se manejaba un modelo de concepción astronómica adecuado a los hechos, es plausible encontrar un asocio de la lluvia de estrellas de 1799 a una aurora boreal. En una carta que dirige Fray José Antonio Liendo y Goicoechea a don José Damas y reproducida en El Mensajero del Clero de 1928 (p. 37), comentó lo siguiente: "Una especie de nube rara, transparente, luminosa que aparece del lado norte en figura de un segmento de círculo, cuya parte occidental es la primera que se ilumina: de este segmento comienzan a salir arcos luminosos, crestones y rayos de una luz muy viva, percibiéndose un movimiento de hervor y confusión de unas partes con otras en toda la masa del fenómeno: quando este se encuentra en sumas de grande crecimiento forma una especie de corona con vivísimos rayos hacia el zenit: aparecen de uno a otro punto otros rayos que figuran espadas y lanzas opuestas unas a otras como si fueran de dos ejércitos encontrados: se ven columnas de vivo fuego de color más hermoso que el del oro: y finalmente este meteoro es de los más deliciosos espectáculos que la naturaleza puede presentar a nuestra vista."

La confusión de este fenómeno con una aurora boreal persistía a finales del siglo XIX, como lo consignó el cartógrafo José María Figueroa en su famoso "Álbum" (Archivo Nacional de Costa Rica, Álbum Figueroa, tomo II, f. 24v): "La aurora Borial [sic] o fuego del cielo como creyeron en aquel tiempo, se dejó ver en el año de 1801 [sic]".

Humboldt divulgó la situación ante la comunidad científica y se dedicó a localizar observadores de este fenómeno en diferentes partes del globo, entre ellas Europa (Soto, 2004). Andrew Ellicott, ingeniero y astrónomo estadounidense e instructor de matemáticas en la Academia Militar de West Point, describió la lluvia de estrellas desde un barco en la Florida el 12 de noviembre de
1799: "Alrededor de las dos en punto de la mañana, me llamaron para ver las estrellas fugaces, el fenómeno era grandioso y atroz, el cielo íntegro parecía que estuviera iluminado con fuegos artificiales, volando hacia una infinidad de direcciones, mientras que yo temía constantemente que alguno de ellos fuera a caer sobre el barco. Continuaron hasta que la luz del sol los apagó. Ese fenómeno se extendió sobre una larga porción de las islas de la India Occidental, y fue observado más al norte como St. Mary, donde se aparecieron tan brillantes como a nosotros".

Tendrían que pasar otros treinta y tres años para que en Costa Rica se volviera a tener noticias de un fenómeno celeste de estas magnitudes, según consta en las fuentes consultadas. Mientras tanto, la ciencia iniciaba su proceso de institucionalización en Costa Rica con la fundación de la Casa de Enseñanza de Santo Tomás en la ciudad de San José, la publicación de los primeros textos científicos y el inicio de las primeras exploraciones de la provincia con el propósito de hallar una ruta hacia el Mar Caribe (Solano \& Díaz, 2005).

\section{LA LLUVIA DE ESTRELLAS DE NOVIEMBRE DE 1833}

Para esta fecha de 1833, la población costarricense se revitalizaba en su dimensión económica. Plasmaba a su vez en el discurso oficial, los conceptos ideológicos del movimiento ilustrado, característicos del pensamiento latinoamericano de la época. Su norte en el progreso y en el cambio fortalecen los cimientos estructurales del estado nacional. Surcaba en el marco colonial la presencia del desarrollo del pensamiento científico y filosófico europeo, funcionaba a nivel institucional, la Casa de Enseñanza de Santo Tomás, regentada por el nicaragüense Rafael Francisco Osejo, versado en letras y ciencias, introduciendo en esa casa de estudios los primeros rudimentos de física y meteorología.

En este mismo período, llegan a nuestra región un conglomerado de empresarios y comerciantes europeos, principalmente británicos, con capitales e intereses en la minería y agricultura. Para este entonces, los habitantes de muchas poblaciones del interior costarricense vivenciaron el 
12 y 13 de noviembre un fenómeno celeste que lo denominaron "Los fuegos fatuos" (ver Anexo 2) y que un articulista que escribió bajo el seudónimo de La Centella, al igual que Liendo al finalizar el siglo anterior, manifestó que era una "aurora boreal" al comparar erróneamente el fenómeno con la descripción ofrecida por Nicolas BoileuDespréaux en una de sus obras (véase Anexo 3). Esta información no fue incluida por Cleto González Víquez en su obra de 1910 “Temblores, terremotos, inundaciones y erupciones volcánicas en Costa Rica, 1608-1910", donde se mencionan varios de los fenómenos celestes analizados en el presente trabajo.

El periódico "El Noticioso Universal" publicó que los fuegos se vieron el mismo día en los poblados del interior del Estado. Estos fuegos, según carta particular de una persona fidedigna, se presenciaron en la ciudad de León, expediendo muchos rayos grandes y continuando posteriormente innumerables pequeños que parecía que incendiaban aquella ciudad. Otro tanto se observó en Chinandega con la circunstancia de haberse antes tendido una nube muy oscura y de haber caído varias luces hasta la tierra, consternando a sus habitantes, quizás por falta de conocimiento de estos fenómenos, a pesar de que ya en 1799 existían registros de una lluvia de estrellas similar a esta.

Páez (1994) sintetiza la experiencia de la manera que se detalla a continuación, pero centrándose en hechos ofrecidos por un documento estadounidense: "ocurre otra lluvia de estrellas en la noche del 12 de noviembre del año de 1833 (idéntica fecha a la del inicio del siglo, [...]), pero de ésta si hay una descripción más detallada. Afortunadamente la lluvia de estrellas á que nos hemos referido, se ofreció á los ojos de todos los habitantes de la América, desde Colombia hasta Alaska. En el boletín oficial de Washington, $\mathrm{N}^{\circ}$ 56, del 28 de febrero de 1834, se lee la descripción siguiente: "Charleston, 14 de noviembre. Fenómeno brillante. En la noche del martes 12 del corriente [noviembre, 1833], la atmósfera tanto en la ciudad como en sus inmediaciones, ha ofrecido un brillante espectáculo de meteoros, estos eran millares de estrellas que caían, de las cuales algunas eran de un tamaño extraordinario, que se lanzaban de todos lados en el espacio celeste como si viniesen a herir la tierra [...] El capitán Jackson, que estaba en el mar, durante la noche, á casi 9 millas de la costa, nos ha dicho que el firmamento había estado toda la noche iluminado con los fuegos resplandecientes de estos meteoros, ofreciendo á la vista un espectáculo á la vez sublime y magnífico, acompañado de uno á otro momento, de un ruido quese asemejaba mucho á la detonación de las armas de fuego de poco calibre.

Flammarion (1883) señala que en territorio americano en 1833, se observó la lluvia de estrellas de Las Leónidas. El científico Denison Olmsted de New Haven (Conecticut, Estados Unidos) analizó la periodicidad del fenómeno ocurrido entre el 12 y 13 de noviembre de cada año y que alcanzó gran notoriedad en dicho año (Weasner, 2001).

Flammarion (1883) comenta: "El período de las grandes apariciones de noviembre indicado por Olbers desde 1837, acababa de confirmarse en 1866 y podia fijarse en 33 años y una fracción, trazando alrededor del Sol una elipse cuyo eje mayor correspondiese á esta duración, encontró que la duración del movimiento de estas estrellas fugaces para recorrer su órbita es de 33 años y 1/4 es decir, la misma que la del cometa de Tempel." El cartógrafo costarricense José María Figueroa (Archivo Nacional de Costa Rica, Álbum de Figueroa, tomo I, f. 37v), comparte esta posición y asocia este notable fenómeno con el paso del cometa Tempel, a raíz de los estudios publicados en la "Cosmografía" de Charles Briot (1817-1882), la "Cosmografía" de Guillermin y el "Cosmos" de Alexander von Humboldt, autor que como se indicó anteriormente, presenció Las Leónidas junto con el naturalista Bonpland en 1799.

Páez (1994) se pregunta: "Sólo nos queda llamar la atención sobre el hecho que sí hubo fenómenos celestes que pudieran haber incentivado a nuestros antepasados por desarrollar un beneplácito por la Astronomía." La respuesta sería afirmativa, ya que en los periódicos de los años siguientes, como "El Mentor Costarricense", se publica una sección titulada las "Afecciones astronómicas" (El Mentor Costarricense, 19-VII-1845:372), continuada por su sucesor, "El Costarricense", publicación dirigida por el Dr. Nazario Toledo (1807-1887), a partir del 21 de noviembre de 1846 (El Costarricense, 21-XI-1846: 5). 
Al mismo tiempo, la llegada de una pléyade de científicos, en su mayoría europeos, contribuyen a la génesis de una incipiente y activa comunidad científica y a la fundación de instituciones como la Junta Económica Itineraria (1843) y el Protomedicato de Costa Rica (1857), que serían las precursoras de las futuras insituciones científicas del país (Solano \& Díaz, 2005).

\section{LA CAÍDA DEL METEORITO DEL $1^{\circ}$ DE ABRIL DE 1857}

Los periódicos de la época siguieron informando de nuevos fenómenos astronómicos al lado de los hechos militares y políticos que generaban controversia en la población. Uno de ellos fue la caída de un meteorito en Heredia, como lo publicó la Crónica de Costa Rica de abril 4 de 1857 (González, 1910), donde apenas se destaca algunas de sus características generales: "El primero de abril, de 7 á 8 de la noche, la capital se vió repentinamente iluminada por un meteoro que cruzó de Sur á Norte lanzando grandes exhalaciones". Y luego, el 9 de mayo de 1857: "El meteoro que alumbró repentinamente la República, el primero de abril pasado, lanzó sobre una de las poblaciones inmediatas á Alajuela, unas grandes piedras negruzcas, metálicas y como de unas cinco ó seis libras de peso, que en vano hemos querido analizar".

Las limitadas descripciones proporcionadas por los medios de la época hacen pensar que a nivel nacional su caída provocó poco impacto, debido al interés con que el gobierno y la sociedad costarricense mantenían sobre el desenlace de la guerra contra las fuerzas filibusteras de William Walker, en esa época acantonadas en Rivas, donde enfrentaron una fuerte resistencia hacia el asedio de las tropas centroamericanas que lo rodeaban y cuya rendición tuvo lugar el 1 de mayo de 1857 (Obregón, 1991; Crónica de Costa Rica, 4-IV-1857, p. 2-3 y 9-V-1857, p. 2-3).

De acuerdo con Soto (1989), la trayectoria del meteorito fue trazada por una bola de fuego que produjo un ruido similar a la de una detonación al hacer contacto con la superficie terrestre. A diferencia de la actitud mostrada por las autoridades locales y la población costarricense en general, la comunidad científica mantuvo un interés por recuperar muestras del meteorito y la mayor llegó a pesar aproximadamente $1 \mathrm{~kg}$. Actualmente, se conservan varias muestras en varias instituciones científicas, incluida la Escuela Centroamericana de Geología de la Universidad de Costa Rica (Soto, 1989; 2004).

El diplomático y estudioso chileno Francisco Solano Astaburuaga, Encargado de Negocios de Chile en Costa Rica, recibió instrucciones de persuadir a las autoridades costarricenses para firmar el Tratado de Unión Americana, suscrito en Santiago por los gobiernos de Chile, Ecuador y Perú (1856) (Archivo Nacional de Costa Rica, Relaciones Exteriores, número 28, expediente 6, ff. 21-27 y expediente 16 f. 31). Este funcionario, interesado en el desarrollo científico de su país, obtuvo una muestra del meteorito y una vez cumplido su objetivo político, retornó a Chile. Una vez instalado en su país, entregó la muestra del meteorito a la colección mineralógica de la Universidad de Chile, siendo estudiada y analizada por el científico polaco Ignacio Domeyko, investigador de los meteoritos hallados en el desierto de Atacama. Domeyko publicó sus resultados en un artículo titulado "Examen y descripción de una aerolita, caída en Costa Rica el primero de Abril de 1857" en los "Anales de la Universidad de Chile" en 1859 (Soto, 1989, 2004; de Bon \& Carvajal, 2004).

Este artículo, enviado en 1858 a la Facultad de Ciencias Físicas y Matemáticas de la Universidad de Chile para su publicación, inicia con una referencia a la obra científica de Humboldt relacionada con el tema y en el análisis se le clasificó como "piedra meteórica". Demuestra el contacto que tuvo el científico con la muestra, la cual comparó con una similar procedente de Francia y destaca de la importancia que tiene para la ciencia estar en contacto con dicho material. Reconoció que en su composición química entran los mismos elementos y minerales conocidos en nuestro planeta.

En 1863, en el listado Büchner se le registra como el meteorito "Heredia" por su lugar de impacto y no sería hasta un siglo después en que Mason (Soto, 2004) lo estudiaría con mayor detalle, catalogándolo como una condrita olivino-broncítica brechiada. Según Soto (2004), es factible que su origen pueda ser el asteroide Hebe, que se encuentra en el cinturón principal de asteroides. 


\section{LAS ESTRELLAS FUGACES Y EL BÓLIDO DE 1896}

Gracias al aporte de una pléyade de científicos, exploradores y estudiosos, principalmente alemanes, entre ellos Karl von Scherzer, Moritz Wagner, Alexander von Frantzius, Karl Hoffmann, Ludwig Daser, Julián Carmiol, Ferdinand Streber, Franz Kurtze y Franz Rohrmoser, se sientan las bases de futuras instituciones científicas como la Oficina de Obras Públicas, la Oficina de Estadística, el Museo Nacional, el Observatorio Meteorológico y el Instituto Físico-Geográfico Nacional (Solano, 1999; León, 2003; Hilje, 2006).

Al finalizar el siglo XIX, el marco institucional existente en el país permite el estudio e investigación de varios fenómenos celestes que ocurren de la época, principalmente estrellas fugaces.

En 1896, las fuentes registran varios fenómenos celestes, en una coyuntura donde varias de las observaciones astronómicas fueron llevadas a cabo por el Ing. Pedro Nolasco Gutiérrez, Jefe de la Sección Meteorológica del Instituto FísicoGeográfico Nacional (fundado en 1889). Este funcionario anotó el paso de varias estrellas fugaces sobre los cielos de la capital, denominándolas con el sinónimo de errantes y "aerolitos". Gutiérrez puso especial interés en las observaciones astronómicas durante el tiempo que ejerció dicho puesto, en la misma época en que el científico suizo Jean Rudin fomentaba el estudio de esta ciencia entre la población costarricense (Páez, 1994; Díaz, 2003; Solano \& Díaz, 2005).

Las estrellas fugaces observadas fueron descritas en el último número de los "Anales del Instituto Físico Geográfico", la principal revista de difusión científica del país, que alcanzó una distribución universal y se detallan en la siguiente lista (Anal. Inst. Fís. Geog. Nac. C. R., 1896, p. 21, 57 y 63; Díaz, 2005):

1) 20 de marzo de 1896, 9:50 PM: desprendimiento de un aerolito con dirección este - oeste, cayendo en la dirección de Alajuelita (suroeste de la capital). En esta localidad se sitúa la hacienda cafetalera La Verbena, en esa época propiedad de la Sociedad Tinoco y Cía., donde se instaló una estación pluviométrica que registró precipitaciones en 1896 y para el período 1898-1905 (Díaz, 2003).
2) 6 de setiembre de 1896: observación de dos estrellas errantes, la primera a las 10:31 p.m., con dirección sureste-noroeste y la segunda a las 10:32: p.m. con dirección noreste-suroeste.

3) 29 de octubre de 1896, 8:37 p.m.: estrella errante que partió de la constelación del Cisne, con dirección a la estrella Vega.

Es presumible que Gutiérrez haya solicitado un informe de las observaciones de estrellas fugaces al Ing. Pedro Reitz, Jefe de la Sección Meteorológica del Instituto Físico Geográfico, relativo a unos reportes de lluvias de estrellas en la ciudad de Cañas a finales de noviembre de 1892. El propósito de dicho informe se debió a que "en los observatorios europeos se había encargado personalmente al señor Reitz, entre otros estudios importantes que convenía hacer en nuestro país, el de esa clase de fenómenos" (La Unión Católica, 8-XII-1892, p. 387).

Aunque Reitz manifestó que en la fecha de las observaciones, el 23 de noviembre, el cielo de San José se encontraba bastante nublado, el articulista consideró que dicho fenómeno no era extraño y más bien, ya científicos como François Arago (1786-1853) quien fue director del Observatorio de París y el barón Alexander von Humboldt habían determinado la periodicidad de varios radiantes a lo largo del año (La Unión Católica, 8XII-1892, p. 387). Dado el interés científico de dicho artículo periodístico, se transcribe completo en el Anexo 4.

Además, en abril de ese mismo año, se observó en Talamanca la caída de un bólido, tal y como lo expresa el siguiente artículo publicado por "La Unión Católica" (1896, p. 438): "De Talamanca comunica á La República el señor don A. Marín J. con fecha 10 del presente lo que sigue: 'El jueves último [9 de abril] que llegué á ésta me encontré con una novedad que pongo en conocimiento del público por si alguien quiere y puede explicar el fenómeno: 'Hacia las 7:30 del domingo 5 del presente [abril], los policiales vieron desde el cuartel y en dirección N.O. elevarse una gran luz semejante á la eléctrica, que iluminó instantáneamente todo el valle. A una altura considerable, que no pudieron apreciar, el globo estalló y se descompuso en partículas encendidas. Dos minutos después se oyó una detonación muy fuerte, atronadora, como la producida por un cañón de muy grueso 
calibre, la cual ocasionó un sensible movimiento oscilatorio, como de temblor, en el suelo, en las casas y en los objetos colgantes.' Cuentan los indios que nunca han visto, oído ni sentido algo semejante."

El reporte del fenómeno observado en Costa Rica debió emitirse en Sipurio, antigua residencia de las autoridades civiles y eclesiásticas en Talamanca, situado en las inmediaciones del actual Amubri (Drüg, 1995). Sipurio fue visitado por miembros de una comisión científica del Instituto Físico-Geográfico en 1894 para estudiar la viabilidad de la navegación del río Telire y corroborar los conocimientos aportados por el geólogo William Gabb en la década de 1870 (Conejo, 1972). En dicho lugar se obtuvieron datos meteorológicos y varios años después, se instaló una estación pluviométrica, que proporcionó datos de 1901 a 1905, cuyo principal observador fue el Presbo. Agustín Blessing Prinsinger, responsable de la misión de Talamanca (1899-1905) y futuro Vicario Apostólico de Limón (Díaz, 2003; Drüg, 1995). Sipurio fue un importante polo de colonización en un territorio fronterizo que tenía una dinámica comercial propia, poco ligada al Valle Central pero bastante vinculada a la economía caribeña (Boza, 2004). Además, en sus inmediaciones se situó la Colonia de San Bernardo.

Las descripciones de observaciones de fenómenos celestes proseguirán en los siguiente años, cuando el Instituto Físico-Geográfico se reestructura tras una breve etapa en que sus funciones fueron suspendidas (1899-1901) a raíz de la crisis económica que afectó al país al finalizar el siglo XIX.

Los reportes de tales observaciones se confeccionaban con base en los testimonios de quienes lograron presenciarlos, muchas veces asociados con fenómenos sísmicos, como ocurrió el 12 de octubre de 1907, cuando varios sismos fueron percibidos en la capital. De acuerdo con Ester Morales Fernández, maestra y encargada de la Sección Meteorológica del Observatorio Nacional, poco antes del anochecer fue observada la caída de un posible meteorito, cuya descripción es un tanto confusa, debido al temor que generaron los sismos de ese día. Morales señala que el pueblo designaba dicho fenómeno celeste bajo el término de "culebrina" (La Prensa Libre, 14-X1907, p. 2): "Varias personas de esta ciudad [San José] aseguran haber visto cruzar en dirección E.O. una claridad viva, que ellas llaman culebrina, un instante inmediato al primer temblor [5:30 de la tarde]. Aseguran muchas personas que al principio de los primeros temblores atravesó un bólido de NW-SE."

Un mes después, en la noche del 6 de noviembre, varios testigos presenciaron la caída de estrellas fugaces, descritas como "brillantes y dejaban á su paso difusos rastros luminosos" (La Prensa Libre, 7-XI-1907, p. 2). ¿Tendría alguna relación con Las Leónidas u otro enjambre similar o más bien se trató de un fenómeno aislado?

Soto (2004) hace un registro de varias observaciones de fenómenos celestes publicadas por periódicos como "El Noticiero" entre 1903 y 1912, en los que se describen posibles lluvias de estrellas, avistamiento de bólidos o caídas de meteoritos. Debido a que solamente hay registro de ellas en documentos periodísticos que presentan informaciones parciales y no siempre detalladas, tales observaciones no se pueden corroborar con otras fuentes de la época y a diferencia de lo sucedido en Heredia (1857), no se rescataron posibles fragmentos.

Sin embargo, será al finalizar la década de 1900 que se va a observar uno de los mayores fenómenos celestes presenciados en el país: el bólido del día en que ocurrió el terremoto de Santa Mónica.

\section{EL BÓLIDO DEL 4 DE MAYO DE 1910}

En la noche del 4 de mayo de 1910, en la misma época en que se observaba el cometa Halley, pocos minutos después del terremoto de Cartago, tuvo lugar el avistamiento de un bólido en territorio costarricense, observado en las poblaciones del Valle Central recién afectados por el movimiento telúrico y que según el parte del buque alemán "Hator" se precipitó en las aguas del Golfo de Nicoya (La Prensa Libre, 1910, p. 2). El cuadro 1 resume algunos de los testimonios conservados de dicha observación. 
Este fenómeno también se conservó en la memoria oral de los habitantes de Cartago y el escritor nacional Carlos Gagini (1865-1925), se refirió a él en su cuento "El tesoro del Coco" como "un enorme globo de fuego [que] surcó el firmamento y fue a sepultarse en las aguas del Golfo de Nicoya" (Soto, 2004). Natwent (1910) publicó en "La Información" haber observado un bólido que surcó los cielos del Valle Central el 28 de setiembre de 1910 y lo calificó como "más grande que el que se vio la noche del 4 de mayo, momentos después del terremoto".

Estos testimonios, a diferencia de los que solían aparecer en la prensa de la época, se ven validados gracias al informe escrito por el naturalista Anastasio Alfaro González, en calidad de Director del Museo Nacional y que fue anexado a la obra "Temblores" del Lic. Cleto González Víquez, recuento cronológico de eventos geofísicos en la historia costarricense desde tiempos coloniales hasta el terremoto de Cartago ocurrido en el año de su publicación en 1910 (Soto, 2004).
Alfaro había recibido indicaciones del geólogo estadounidense Thomas A. Jaggar, fundador del Observatorio Volcanológico de Hawai, para imprimir cuestionarios para registrar el impacto del terremoto en la población y en el que se señala un apartado denominado "Iluminación posterior" que se refiere al aerolito: "Hay igualmente gran número de observadores que aseguran haber visto un bólido, que pasó de Esta á Oeste. Sobre la cordillera volcánica, desde el Turrialba hasta atravesar la península de Nicoya, siguiendo, poco más o menos, el paralelo $10^{\circ}$ Latitud Norte. Con respecto á la hora, varía mucho pero todos están de acuerdo en que pasó rápidamente, poco después del terremoto, algunos estimaron el intervalo, entre uno y otro fenómeno, en segundos solamente, pero en otros dicen que fué un cuarto de hora más tarde ó veinte minutos."

Solo el reporte del barco Hator indica su caída en el océano, si bien no hay reportes de daños causados por la alteración del oleaje en las poblaciones vecinas del Golfo de Nicoya. Los meteoritos

\section{Cuadro 1}

Observaciones del bólido del miércoles 4 de mayo de 1910

\begin{tabular}{|c|c|c|}
\hline $\begin{array}{l}\text { Estado del tiempo y lugar } \\
\text { de las observaciones }\end{array}$ & Hechos observados & Reacción del pueblo y autoridades \\
\hline No indicados (San José) & $\begin{array}{l}\text { "Vi un bólido que iluminó el horizonte }[\ldots] \text { El } \\
\text { bólido caminó del centro del cielo en direc- } \\
\text { ción noroeste [...] Como } 5 \text { minutos después } \\
\text { [del sismo]" }\end{array}$ & $\begin{array}{l}\text { Observador: Francisco Jiménez Núñez (1861- } \\
\text { 1938), farmacéutico. Anotó la observación en } \\
\text { una fórmula distribuida por el Museo Nacional } \\
\text { [véase infra] para tener un registro de los efec- } \\
\text { tos del sismo en el país, fechada en San José el } \\
15 \text { de junio de } 1910 \text {. }\end{array}$ \\
\hline $\begin{array}{l}\text { Llovizna posterior al ter- } \\
\text { remoto (Cartago). }\end{array}$ & $\begin{array}{l}\text { "En aquellos momentos atravesó el espacio desde } \\
\text { el zenit al Oeste una ráfaga de fuego y un peque- } \\
\text { ño globo luminoso en que se deshizo, produjo un } \\
\text { ruido como el de una palmada lejana." }\end{array}$ & $\begin{array}{l}\text { Observador: Alfonso Pérez Martín. Profesor es- } \\
\text { pañol que fungía en esa época como director del } \\
\text { Liceo de Costa Rica (1907-1913). Se encontra- } \\
\text { ba en Cartago durante la noche del terremoto. }\end{array}$ \\
\hline $\begin{array}{l}\text { Mal tiempo (Golfo de } \\
\text { Nicoya). }\end{array}$ & $\begin{array}{l}\text { “... vieron iluminarse de improviso, con toda la } \\
\text { claridad del medio día dicho Golfo, percibién- } \\
\text { dose claramente las islas todas. Miraron y vieron } \\
\text { al gran viajero dijéramos de Manzanillo á Bocas } \\
\text { del Toro, y luego se escuchó un grande y extraño } \\
\text { sonido, y poco después se produjo en las aguas } \\
\text { una serie de ondas tan fuertes, que amenazaba á } \\
\text { zozobrar la nave que los conducía”. }\end{array}$ & $\begin{array}{l}\text { Observadores: Mr. Smith, Mr. Krieguer y Aure- } \\
\text { lio Castro. Tripulantes del buque Hator. }\end{array}$ \\
\hline
\end{tabular}

Fuentes: Arch. Mus. Nac. C. R., Exp. 8566 (1910); Arch. Nac. C. R., Fidel Tristán. Exp. 49 (1910); Pérez et al. (1911), La Prensa Libre (1938) y Zamora \& López, 1988, p. 79-80. 
son en algunas ocasiones, la causa de un tsunami, pero si no causó mayores problemas, ha de haber sido un meteorito de pequeñas dimensiones.

\section{CONCLUSIONES}

A lo largo del período de estudio, se analizó la importancia que tuvo para la ciencia y la cultura costarricenses, las observaciones de fenómenos celestes como los meteoritos, bólidos y estrellas fugaces. Su presencia está documentada en fuentes coloniales y decimonónicas, donde se busca una explicación racional del fenómeno por parte de las autoridades coloniales y los estudiosos de la época, mientras que paralelamente, se recurría a prácticas religiosas para evitar sus consecuencias negativas, como ocurrió con las lluvias de estrellas de Las Leónidas en 1799 observadas en Cartago y Tobosi y en 1833 reportadas en varios pueblos del Valle Central.

Las Leónidas de 1799 fueron atribuidos tanto a una posible manifestación volcánica como a la supuesta presencia de una aurora boreal, no obstante su errónea atribución, se insistía en el carácter natural del fenómeno. Se generó un debate al nivel de la naciente prensa costarricense (que incluyó temas de índole científico), por ejemplo, la que ofrece $\mathrm{La}$ Centella, quien intenta hallar una respuesta en una de las obras de Despréaux al comparar sus observaciones con la descripción de una aurora boreal.

Los fenómenos posteriores fueron de mayor interés para la ciencia, principalmente la recolección de muestras del meteorito de 1857 , caído en Heredia, las cuales se encuentran esparcidas por América y Europa y cuyo análisis maravilló a un hombre de ciencia como Domeyko, iniciador del estudio científico de los meteoritos en Chile, quien compara la muestra de este objeto con una similar procedente de Francia. La muestra procedente de Costa Rica fue obtenida por el diplomático Francisco Solano Astaburuaga, quien cumplió una misión para el gobierno chileno mientras los ejércitos centroamericanos combatían las fuerzas filibusteras de Walker en Nicaragua, por lo que se puede inferir que la atención brindada al conflicto bélico por parte de la prensa puso al meteorito en un plano secundario para los lectores costarricenses.

Los procesos de institucionalización de la ciencia en Costa Rica que propiciaron el establecimiento de un "régimen de cientificidad" (Viales \& Clare, 2006; 2007) donde confluían varios factores económicos, políticos, sociales y culturales que permitieron el florecimiento de instituciones y prácticas científicas hicieron posible que estos fenómenos fueran registrados, principalmente por los funcionarios del Instituto Físico-Geográfico Nacional, quienes consignaron datos de algunos de ellos mientras efectuaban observaciones meteorológicas y entre los que merecen citarse grandes estrellas fugaces observadas en 1896 por el Ing. Pedro Nolasco Gutiérrez y las de 1907 por la maestra Ester Morales en el Valle Central. Para zonas distantes de los centros urbanos, se recurría a los reportes de las autoridades locales, como ocurrió con el bólido observado en Talamanca en 1896.

Finalmente, la aparición de un bólido en el cielo costarricense, minutos después de haberse estremecido el territorio nacional como consecuencia del terremoto del 4 de mayo de 1910, debió haber producido pánico, más en una época en que todavía se atribuían estos fenómenos a manifestaciones sobrenaturales. Es preciso recordar que dicho terremoto fue atribuido por el Ing. Pedro Nolasco Gutiérrez al paso del cometa Halley, visible en esa misma época, lo que acrecentó aun más el temor hacia los fenómenos celestes entre las clases populares del país pese a las advertencias hechas por el Dr. Jean Rudin del carácter inofensivo de tales objetos celestes (Molina, 1994).

\section{AGRADECIMIENTOS}

Al Ing. Rolando Arias Herrera por su colaboración en el trazado y representación de los fenómenos celestes analizados en el mapa que acompaña el presente artículo. A Giovanni Peraldo y Gerardo J. Soto por su revisión crítica. La presente investigación es producto del Proyecto 
Meteorología e Impacto Social Ambiental en Centro América y México (VI-805-97-519) y del Programa Estudios Sociales de la Ciencia, la Técnica y el Medio Ambiente (VI-805-A4-906), ambos del Centro de Investigaciones Geofísicas de la Universidad de Costa Rica.

\section{REFERENCIAS}

BOZA, A., 2004: Indígenas, comerciantes, transnacionales y estados: población, comercio y política entre las poblaciones indígenas de la Gran Talamanca, Costa Rica (18401930): (Chirripó, Estrella, Talamanca y Pacífico Sur).- 371 págs. Universidad de Costa Rica, San José [Tesis M.Sc.].

CHACÓN, L.A., 1967: Don Diego de la Haya Fernández.- 276 págs. ECR. San José.

CONEJO, A., 1972: Materiales para una biobibliografía costarricense del Dr. Henri Pittier Dormond.- 657 págs. Universidad de Costa Rica, San José [Tesis Lic.].

DE BON, C.C. \& CARVAJAL, R.A., 2004: Ignacio Domeyko, precursor del estudio de los meteoritos en Chile (I Parte).- Bol. Min. 1164. SONAMI. Santiago, Chile. http://www.sonami.cl/cgi-bin/procesa. pl?plantilla=/boletinmensual_detalle. html\&id_art=542.

DÍAZ, R.E., 2003: El proceso de institucionalización de la meteorología en Costa Rica (1887-1949).- 383 págs. Universidad de Costa Rica, San José [Tesis Lic.].

DÍAZ, R.E., 2005: "Los costarricenses no han dejado de admirar las magnificencias del cielo": La evolución de las ideas astronómicas en Costa Rica (1814-1910).- Diálogos, Revista Electrónica de Historia, 6 (1), enero-agosto. <http://historia.fcs.ucr.ac.cr/articulos/2005/ideas_astro-rdiaz.pdf>.
DRÜG, B., 1995: Congregación de la Misión. Historia de la Viceprovincia de Costa Rica. 1873-1993.- 116 págs. Ediciones Fe y Vida. Teruel, España.

FLAMMARION, C., 1883: La atmósfera. Descripción de los grandes fenómenos de la naturaleza. I. Barinaga y Corradi, L. (Tr.).- 471 págs. Imprenta y Librería de J. Gaspar, España.

FONT-TULLOT, I., 1986: Historia del clima de España, Cambios climáticos y sus causas.- Instituto de Transportes, Turismo y Comunicaciones, España.

GONZÁLEZ FLORES, L.F., 1976: Historia de la influencia extranjera en el desenvolvimiento educacional y científico en Costa Rica.- 296 págs. ECR, San José.

GONZÁLEZ VÍQUEZ, C., 1910: Temblores, Terremoto, Inundaciones y Erupciones Volcánicas en Costa Rica 1608-1910.- 200 págs. Tipografía de Avelino Alsina, San José.

HARDY, R., WRIGHT, P., GRIBBIN, J. \& KINGTON, J., 1983: El libro del clima. Rubio, A. M. (Tr.).- 223 págs. Hermann Blume Ediciones, España.

HILJE, L., 2006: Karl Hoffmann: naturalista, médico y héroe nacional.- 200 págs. INBio, Heredia.

LEIVA, R., 1980: Vigencia del sabio Del Valle.442 págs. EDUCA, San José.

LEÓN, J., 2003: La exploración botánica de Costa Rica en el siglo XIX.- En: PERALDO, G. (comp.): Ciencia y Técnica en la Costa Rica del siglo XIX.- Editorial Tecnológica. Cartago: 129-186.

MELÉNDEZ, C., 1974: La Ilustración en el antiguo Reino de Guatemala [2 ${ }^{\mathrm{a}}$ ed.].- 218 págs. EDUCA. San José, Costa Rica. 
MOLINA, I., 1994: El paso del cometa Halley por la cultura costarricense de 1910.- En: MOLINA, I. \& PALMER S. (eds.): El paso del cometa. Estado, política social y culturas populares en Costa Rica (1800-1950).Ed. Porvenir \& Plumsock Mesoamerican Studies, San José: 167-190.

NATWENT, O., 1910: Fenómeno atmosférico. La Información, 30 de setiembre: 2 .

OBREGÓN, R., 1991: Costa Rica y la guerra contra los filibusteros.- 410 págs. Mus. Hist. Cult. Juan Santamaría, Alajuela.

PÁEZ, J., 1994: Aspectos históricos de la astronomía en Costa Rica. Ciencia y Tecnología, 18(1-2): 51-73.

PEREIRA, G., s.f. Los guaraníes y las leónidas. $<$ http://www.umsanet.edu.bo/org/astro/etnopub.htm> [Consulta: 2005].

PÉREZ, A., VALLADARES, R., CASTRO, O., BARRIONUEVO, J. \& ESQUIVEL DE LA GUARDIA, A., 1911: Rafael Ángel Troyo. Ofrenda y Homenaje.- 83 págs. Imprenta del Comercio, San José.

SOLANO, F., 1999: El proceso de institucionalización de la meteorología en Costa Rica en el siglo XIX.- 227 págs. Universidad de Costa Rica, San José [Tesis Lic.].

SOLANO, F. \& DÍAZ, R., 2005: La ciencia en Costa Rica: (1814-1914). Una mirada desde la óptica universal, latinoamericana y costarricense.- 70 págs. ECR, San José.

SOTO, G.J, 1989: El meteorito tico - Semanario Universidad. 861, 3 de marzo: 6.

SOTO, G.J., 2004: Meteoritos y meteoros en Costa Rica (verdaderos, posibles y falsos).Rev. Geol.. Amér. Central, 31: 7-23.
VIALES, R.\& CLARE, P.; 2006-2007: El Estado, lo transnacional y la construcción de comunidades científicas en la Costa Rica liberal (1870-1930). La construcción de un 'régimen de cientificidad'.- Diálogos, Revista Electrónica de Historia, 6 (2). setiembrefebrero: 145-168. <http://www.ts.ucr. ac.cr/ historia/articulos/2006/vol2/6-rviales_clare.pdf>.

WEASNER, M.L., 2001: Observing meteors - The leonids. <http://www.weasner.com/ etx/ref_guides/leonids01.html>.

ZAMORA, C.M. \& LÓPEZ, B., 1988: Liceo de Costa Rica: un siglo de existencia.- 167 págs. Com. Nac. de Conm. Hist., San José.

\section{Fuentes hemerográficas y de archivos}

An. Inst. Fís. Geog. Nac. C. R., 1896: Tomo IX.- Tipografía Nacional. San José, Costa Rica.

Arch. Mus. Nac. C. R.. Inventario General de Bienes. Exp. 8566 (1910).

Arch. Hist. Arq. Mons. Bern. Aug. Th. Hoff. Sección Gobierno Eclesiástico. Serie Visitas Pastorales. 4 (1882-1899).

Arch. Nac. C. R. Álbum de Figueroa. I y II.

Arch. Nac. C. R. Fidel Tristán. 2. Exp. 49 (1910).

Arch. Nac. C. R. Relaciones Exteriores. 28. Exp. 6. Chile (1857) y Exp. 16. Funcionarios y Particulares (1857).

El Mensajero del Clero. 1928,.- Febrero: $37-$ 38, XL (2). Imprenta Lehmann. San José, Costa Rica. "CARTA DE FRAY. JOSÉ ANTONIO GOICOECHEA AL MUY ILUSTRE DON JOSÉ DAMAS".

“DONDE CAYÓ EL GRAN METEORITO”. 1910. La Prensa Libre. 14 de mayo:2.

El Costarricense. 1846. 21 de noviembre: 5.

El Mentor Costarricense. 1845. 19 de julio: 372.

El Noticioso Universal. 1833.15 noviembre: 412.

El Noticioso Universal. 1833. 6 de diciembre: 438 y $442-444$.

El Noticioso Universal. 1833.29 de diciembre: 486. 
“FENÓMENO CURIOSO”. 1896. La Unión Católica. 21 de abril: 438.

"FENÓMENOS ESTELARES". 1907. La

Prensa Libre. 7 de noviembre: 2.

La Crónica de Costa Rica. 1857. 4 de abril: 2-3.

La Crónica de Costa Rica. 1857. 9 de mayo: 2-3.

La Información. 1910. 30 de setiembre: 2.

La Prensa Libre. 1938. 13 de octubre: 4.

La Unión Católica. 1892. 8 de diciembre de 1892:387.

“LOS TEMBLORES DEL SÁBADO.” 1907. La Prensa Libre. 14 de octubre: 2.

\section{ANEXOS}

\section{Anexo 1: Lluvia de estrellas de las Leónidas de 11-12 de noviembre de 1799}

\section{Lluvia de fuego}

El Gobernador de Costa Rica don Tomás de Acosta, el 20 de noviembre de 1799 dirige carta al Capitán General Domás y Valle acerca del fenómeno que observó:

"La noche del día once de este, [noviembre de 1799] en que finalizaron las pláticas hechas felizmente por Fray Manuel de la Fuente, religioso Recoleto del Colegio de Cristo, de esa ciudad, acaeció en esta uno de los más portentosos fenómenos que se han visto en este hemisfero y tal vez. en el mundo:-este fue que hallándose el tiempo sereno, la luna clara, el cielo despejado y sólo cerrazón de nubes en el primero y segundo cuadrante, aunque á mucha distancia, se vieron á primera noche varias exhalaciones de regular tamaño y proporcionada luz, que corrían del Noreste al Suroeste, que era el viento que soplaba, aunque poco; pero estas exhalaciones se fueron aumentando con la noche en tanto número y tamaño, que á las dos o tres de la mañana eran ya de instante en instante sin guardar dirección fija, sino que indistintamente giraban á todos los vientos, de modo que parecía llover fuego. Muchas eran de tal magnitud que parecían globos de fuego, ó carcaces encendidos, y arrojaban tan copiosas y gruesas chispas que caían algunas hasta el suelo y dejaban en la atmósfera una señal tan larga y ancha que parecía abrirse el cielo, quedando la señal estampada por más de diez segundos. De estas señales unas eran rectas é imitaban la cola ó rabo de los cometas; otras que formaban sinuosidades dejaban el rastro de una serpiente, y las más no dejaban sino la de una exhalación ordinaria; pero por la misericordia divina todas se apagaban en el aire, y sólo he sabido de una que cayó en el pueblo de los indios de Tobosi, como á una legua de esta ciudad, la cual dicen estos ardió largo rato en gruesa llama y á proporción que esta disminuía tomaba un color azul.

Todo este vecindario estaba en pie, y aunque en mi concepto tuve y tengo este acontecimiento por efecto natural, con todo como los ánimos se hallaban dispuestos á penitencia con los muchos sermones que se acaban de hacer, este accidente, á la verdad asombroso, los compungió más y acudiendo al santuario de Nuestra Señora de los Angeles, imagen aparecida y milagrosa patrona de esta ciudad, fue conducida procesionalmente á la Parroquia, como á las cinco de la mañana del día doce, habiéndosele dicho antes muchas misas en su Santuario y después en la Parroquia. Desde que aquel sagrado simulacro salió de su templo, cuando empezaba el día, empezaron también a disminuir las exhalaciones, que totalmente cesaron ó se dejaron de ver con la salida del sol, que fue muy claro todo aquél y los siguientes hasta hoy, sin que en este intervalo se haya notado otra cosa que mantenerse la brisa fresca y recia.

Con motivo de hallarse situada esta ciudad entre varios volcanes, de los cuales los más recelosos son el de Cartago que le queda al Norte, distante como dos leguas, el de Turrialba al Noreste á distancia como de ocho leguas, y el del Dragón al Suroeste como á igual distancia, tuve á bien mandar personas de satisfacción que inmediatamente fueran á reconocerlos con prolijo examen para ver si humeaban, si se les advertía alguna inflamación, si tenían alguna grieta, si se les oía 
algún susurro, si exhalaban algún olor sulfureo o bituminoso, ó si se encontraba en ellos novedad particular; mas regresados los exploradores aseguraban no haber observado cosa alguna extraña.

En vista, pues, de todo lo relacionado y de la general consternación, recelosos de alguna erupción, se prosigue implorando la misericordia y auxilios del Todo-Poderoso por medio de las continuas confesiones, procesiones de penitencia y sermones que sigue predicando con notoria y ejemplar utilidad el citado fray Manuel de la Fuente, por súplica de este celoso cura y Vicario, á cuyo nombre, el mío y el de este vecindario me intereso con V.S.M.I., para que este varón apostólico sea destinado á la reducción de Orosi para que lo tengamos á la mano para nuestro espiritual consuelo en los casos que ocurran, sin que se lo estorbe su Prelado.

Sin embargo de que, como llevo dicho, atribuyo á efecto natural este fenómeno, respecto á los volcanes que nos rodean y muchos minerales que incontestablemente hay en estos contornos, reflexiono también, fundado en aquellas premisas, que cualquiera que sea la causa productiva de estos efluvios inflamables, es muy de temer una erupción, porque en vista de la abundancia que hubo de ellos y lo cargado que estaban de materia eléctrica, considero que el paraje que los arrojó, sea el que fuere, sobreabunda de aquella materia, y por consiguiente muy expuesto á reventar. Yo puedo estar muy errado en mi juicio, porque no he hecho estudio de física experimental, pero de todos modos creo de mi obligación participar á Usted lo acaecido y expresarle lo que siento, por si hubiere alguna cosa que prevenir, y asegurarle al mismo tiempo que no hay lengua, pluma ni pincel capaz de pintar debidamente lo que sucedió la noche del once al doce del corriente, y que la expresión de que llovió fuego sólo se aparta de la realidad en que no cayó en tierra todo el que se desprendió de la atmósfera.-Cartago, 20 de noviembre de 1799.-Tomás de Acosta

Fuente: González Víquez (1910)

\section{Anexo 2: Lluvia de estrellas de las Leónidas del 13 de noviembre de 1833}

Tomado del periódico El Noticioso Universal de, 15 de noviembre de 1833, Nº 46, pág. 412.

\section{Fuegos fatuos}

La madrugada del 13 del presente mes, cómo a las tres, se observó en todos los pueblos del interior los fuegos fatuos que duraron hasta las seis de la mañana, haciéndose visibles á esta hora ya por el rastro color de leche que se dejaba ver ó porque la magnitud y viveza de la luz de algunos se presentaba visiblemente. Este fenómeno fue precedido en la tarde y noche del día anterior, por un recio viento de O.S.O. que duró hasta la expresada hora en que se verificó una revolución causada por una correntada de E.N.E. á la temperatura de $65^{\circ}$ de Fahrenheit.

Estos fuegos (que algunos confuden con la aurora boreal) son los mismos que vieron en Cartago el año de 1800 puntualmente en el mismo día, que han llenado de terror á las gentes sencillas y servido de diversión á las personas de discernimiento. Algunos de estos los estiman como un medio para limpiar la atmósfera y librar á los vivientes de la influencia nociva de las exhalaciones sulfúreas y betuminosas de que estaba cargada aquélla.-EE

Entre otros errores que contiene el párrafo anterior, se nota el de tomar el año de 1800 por 1799, según hemos visto antes.

\section{Anexo 3: Debate acerca de la lluvia de estrellas de 1833}

"CC.EE habla sobre el poder divino y que el firmamento anuncia las obras:

"habla sobre los milagros V.g. la aurora boreal que vimos el martes por la noche día 12 hasta amanecer el día 13 de este mes. Es un fenómeno natural pero que a los ojos del entendimiento se 
descubren grandes dificultades para investigar su naturaleza; ó la causa de donde proviene, y la aurora boreal, es un hecho tan sensible, y palpables á los sentidos de todos los que salimos a ver el cielo. Nó necesitamos mas que de verlo para saber que hubo aurora boreal y se trasmitirá de generación en generación, sin poder ser desmentidos de aquí a dos mil años. $Y$ ¿qué es aurora boreal? Respondo, en satisfacción copiaré la explicación de ella hace Mr. Despreaux en el tomo 5 de su obra titulada Lecciones de la naturaleza.

\section{Aurora boreal}

"Entre todos los fenómenos nocturnos, no hay otro mas notable, y aun á veces mas resplandeciente que la aurora boreal. En el invierno, y hacia el equinoccio de la primavera quando el Cielo esta sereno y la luna tiene poca claridad, se vé con frequencia por la parte del norte una especie de nuves [sic] transparentes, luminosas $y$ de varios colores. Una luz brillante, se comunica consecutivamente á otras nuves, de donde salen, en fin unas ráfagas de luz blanquecina que se estiende [sic] hasta cerca del cenit. Tal es el fenómeno que llamamos aurora boreal. Este meteoro, no está siempre acompañado de las mismas circunstancias. Por lo comun solo hacia la media noche se vé una claridad parecida al alba del día: otras veces se observan tambien surcos y ráfagas de luz, nuves blancas y luminosas que están en un movimiento continuo. Mas quando la aurora boreal debe mostrar todo su esplendor se vé casi siempre en tiempo calmado, y sereno, un espacio obscuro, una nuve negra y densa, cuya orilla superior está rodeada de una banda blanca, y luminosa, de la qual salen muy presto rayos, ráfagas brillantes, columnas resplandecientes que elevandose por instantes toman colores amarillos, y roxos [sic], luego se acercan, y toman nuves luminosas y densas, terminándose por ultimo en coronas blancas, asules de color de fuego, ó de la mas bella purpura que despiden continuamente ráfagas de luz. Este brillante fenómeno aunque tan visible es uno de aquellos efectos naturales, cuya causa no ha podido determinarse todavía con exactitud. Algunos Físicos afirmaron que debia su formación á exhalaciones nitrosas, bituminosas, y sulfureas. Otros le atribuyen á la reflexion y refraccion de los rayos del sol en las nuves y nuves eladas [sic] del Norte, y otros tambien á las porciones desprendidas de la atmósfera que se supone alrededor del Sol, y que mezcladas con las de la tierra fermentan con ella. Igualmente se dá por causa de la aurora boreal, cierta especia de exhalaciones desprendidas del seno de las tierras septentrionales: exhalaciones de una naturaleza bastante parecida á la del fósforo, que reuniendo la luz con el fuego tiene mucho menos fuego que luz. Los fenómenos que acompañan los meteoros ígneos dieron margen á un celebre químico para creer que hay en la parte superior de la atmósfera una capa de fluido inflamable mas ligero que el aire, y que en el punto de contacto de estas dos capas, es donde se efectúan así los fenómenos de la aurora boreal como los de otros meteoros ígneos.

Por lo demas, la misma incertidumbre en que están los hombres mas ilustrados acerca de las causas de la aurora boreal es una nueva, entre otras muchas de lo limitado de nuestra capacidad. Mil cosas de poca consideración confunden muchas á los mas Sabios en sus meditaciones, y se ocultan á todas las investigaciones: tambien hay una multitud de objetos que aun quando reconocemos estar dispuestos con mucha sabiduría y ser utiles, rara vez llegamos a descubrir, sus verdaderos principios, su union con el mundo corpóreo, y con sus diversas partes. Por fortuna esa incertidumbre no influye en nuestra felicidad; pues aunque no podamos determinar exactamente de donde procede la aurora boreal no por eso dejamos de vivir tranquilos y contentos. Sabemos á lo menos, que todos los fenómenos del mundo asífisico como intelectual, no suceden sino por la voluntad de un Señor infinitamente sabio, poderoso, y bueno que los encamina al bien del universo ¡Ah! ¡esto sin duda es bastante para exitarnos á 
adorar al que sabe obrar cosas tan maravillosas, tan superiores á nuestro entendimiento; __ Pero debo aun bendeciros ó Dios mio, por que no me hicisteis nacer en aquellos tiempos de ignorancia, y superticion, en que estos fenómenos llenaban á pueblos enteros de consternación, y de terror. Un espectaculo tan magnifico e interesante solo ofrecía á su turbada imaginación, exercitos y combates que se daban en el aire, y sacaban de ellos los mas funestos pronosticos. La aurora boreal era para ellos un Profeta que les anunciaba yá la guerra, yá el hambre, y yá enfermedades epidémicas. Mas por el contrario, yo hallo en el apacible, y majestuoso brillo de esta luz, una señal de poder, $y$ de la bondad del Altísimo. Veo sin miedo estos fuegos, por que sé que el Señor del Cielo nada há criado para desgracia y tormento de sus criaturas, y puede ser que, en los países septentrionales saquen los hombres de estos fenómenos que tanto poco influjo tienen en nuestras regiones, ventajas que sean para ellos nuevos motivos de reconocer un padre en el autor de la naturaleza.

Hasta aquí el citado autor. La Centella.

Fuente: El Noticioso Universal (1833, p. 438 y 442-444).

\section{Anexo 4: Informe de una lluvia de estrellas en noviembre de 1892}

De Las Cañas nos dicen con fecha 25 de Noviembre último: "El 23 del psesente [sic], por la noche, varias personas vimos el fenómeno más bello de la naturaleza que jamas [sic] viéramos antes. Desde las 7 de la noche se veía en toda la bóveda celeste y en todas direcciones de instante á instante deslizarse como estrellitas pequeñas, dejando varias de ellas tras sí estelas: á veces se deslizaban casi á la par, unas se desprendían de la parte más baja ó inferior." Nuestro corresponsal nos manifiesta además que este fenómeno se prolongó tarde de la noche, que él lo observó hasta las 11 1/2, hora en que se recogió á dormir.

Sabiendo un miembro de nuestra sociedad [¿Pedro Nolasco Gutiérrez?] que en los observatorios europeos se había encargado personalmente al señor [Pedro] Reitz, entre otros estudios importantes que convenía hacer en nuestro país, el de esa clase de fenómenos, dicho señor anotó que en el mes pasado había visto una estrella errante el día 7 [de noviembre] á las 6 h.50 m.p. m. que escribió [sic] una trayectoria desde Júpiter hasta Beta del Pegaso, y otra que el 29 [de noviembre] á 7 h. 31 m. p. m. partía de la zona de la vía láctea [próximamente de Beta del Cisne] y desapareció hacia la bella estrella de lira [Wega: (sic)] La misma persona de estas anotaciones preguntó en nuestro Observatorio si alli se había observado algún fenómeno extraño en el día y momento que acontecía el de Las Cañas , y el señor Reitz tuvo la amabilidad de manifestar que en ese instante no se podía observar el que hubiera, por hallarse el cielo en sus siete décimas partes oscuro. De San Vicente y de Paraca [sic] nos dicen que en esos dos lugares se vieron algunas estrellas errantes el día 23 indicado.

Este fenómeno hoy no es raro desde que Arago, el primero, señaló su periodicidad, que después Humboldt fija para el 22-25 de Abril, 17 de Julio, 10 de Agosto, 12-14-27-29 de Noviembre y 6-12 de Diciembre.

Como su aparición, según dice el último, en el tomo I de su Cosmos, página 112, va acompañada frecuentemente de otros fenómenos meteorológicos, damos cuenta de él.

Daremos más tarde á conocer á nuestros lectores la explicación que sobre esos fenómenos han dado algunos astrónomos.

Fuente: La Unión Católica (1892, p. 387). 